\title{
A Brief History of the Study of Nearby Young Moving Groups and Their Members
}

\author{
Joel H. Kastner \\ Laboratory for Multiwavelength Astrophysics, Center for Imaging Science, and School of \\ Physics and Astronomy, Rochester Institute of Technology; \\ email: jhk@cis.rit.edu
}

\begin{abstract}
Beginning with the enigmatic (and now emblematic) TW Hya, the scutiny of individual stars and star-disk systems has both motivated and benefitted from the identification of nearby young moving groups (NYMGs). I briefly outline the emergence of this relatively new subfield of astronomy over the past two decades, and offer a few examples illustrating how the study of NYMGs and their members enables unique investigations of pre-main sequence stellar evolution, evolved protoplanetary disks, and young exoplanets.
\end{abstract}

Keywords. protoplanetary disks, stars: pre-main sequence, stars: individual (TW Hya, V4046 Sgr, HR 4796, $\beta$ Pic)

\section{TW Hya: a Classical T Tauri Star without a Birthplace}

The identification and investigation of nearby young moving groups (NYMGs) - i.e., loose associations of stars that lie within $\sim 100 \mathrm{pc}$ of Earth and have ages up to $\sim 100 \mathrm{Myr}$ - arguably began with the identification of the late-type, emission-line field star TW Hydrae as a "T Tauri star far from any dark cloud" (Rucinski \& Krautter 1983). This comprehensive study by Rucinski \& Krautter establishing the classical T Tauri nature of TW Hya was motivated by the work of Henize (1976) and Herbig (1978), and led directly to the identification of additional examples of "isolated T Tauri stars" (de la Reza et al. 1989; Gregorio-Hetem et al. 1992). These studies yielded the identification of a literal handful of late-type field stars with T Tauri-like characteristics — in particular, deep Li absorption and apparent mid-infrared excesses $\dagger$ - in the general vicinity of TW Hya.

In their landmark paper, de la Reza et al. (1989) speculated on the potential nature of these lonely T Tauri stars, suggesting they were "kinematically related" and likely formed in situ. Furthermore, on the basis of these stars' high galactic latitudes, de la Reza et al. suggested (without assigning a specific distance range) that TW Hya and its apparent cohort may be relatively close to Earth. The last assertion seemed particularly intriguing, given the potential for "close-up" studies of planetary systems in formation that might be afforded by these and other "isolated" T Tauri stars. Following up on this hunch, Zuckerman et al. (1995) established that the thermal infrared excess from dust around TW Hya (and a few other "isolated," young, IR-excess stars) was accompanied by the presence of residual orbiting molecular (CO) gas, suggestive of the recent cessation of an epoch of planet formation. But the distance and age of TW Hya remained very poorly constrained.

$\dagger$ It is fascinating to note, in retrospect, that two of the five original members of the TW Hya Association were only observed in $\mathrm{H} \alpha$ and $\mathrm{Li}$, and hence identified as young, by de la Reza et al. (1989) and Gregorio-Hetem et al. (1992) thanks to chance alignments with IR-bright background galaxies. 
With the release of the ROSAT (X-ray) All-Sky Survey (RASS), we could address these unknowns. The RASS X-ray data firmly established the youth of TW Hya and its (four) nearby siblings, yet also suggested the five were older than typical of T Tauri stars in dark clouds; we guesstimated (sic) the ages of the five stars as $\sim 20$ Myr (Kastner et al. 1997). With this age estimate, we could then place firmer constraints on the stars' distances, and determined that they were a mere $\sim 40-60 \mathrm{pc}$ from Earth. This range was promptly confirmed by the newly released Hipparcos parallax distances to TW Hya $(D=54 \mathrm{pc})$ and one of its four siblings, HD $98800(D=45 \mathrm{pc})$. In light of their similar ages, distances, and kinematics, we took the (perhaps unwise) leap of christening this little ragtag group of young stars the TW Hya Association (TWA), and declared the TWA "the nearest region of recent star formation" (Kastner et al. 1997).

Fortunately for us, many subsequent studies — beginning with Webb et al. (1999), and extending to the work of Murphy et al. presented at this meeting - have dramatically expanded the TWA's known membership, have confirmed its mean distance as $\sim 50 \mathrm{pc}$, and have honed estimates of its age, which is now generally accepted as $\sim 8$ Myr $\dagger$. Meanwhile, TW Hya itself has gone on to become the Crab Nebula $\ddagger$ of late-stage pre-main sequence accretion and protoplanetary disk studies, with nearly 1000 simbad references (95\% of them since 1997) as of the writing of this review.

\section{The (young association) link between the TWA and $\beta$ Pic}

Not long after the naming of the TWA, Jura et al. (1998) noted that the A stars HR 4796A, $\beta$ Pic, and 49 Cet - all massive debris disk hosts - were underluminous for their colors relative to the field A star population, suggesting all three are young. The youth of the HR 4796AB system had already been established by Stauffer et al. (1995) on the basis of the position of the M-type star HR 4796B above the main sequence. Based on the (relative) sky proximity of $\mathrm{HR} 4796 \mathrm{AB}$ to the original five stars of the TWA, Jura et al. (1998) further speculated that HR 4796A may also be a TWA member (an assertion subsequently confirmed by Webb et al. 1999).

A strong implication of the Jura et al. (1998) analysis was that $\beta$ Pic and the stars of the TWA, though not kinematically or spatially associated, were similarly young. At the time, this was somewhat of a revelation, given that some previous estimates had put the age of $\beta$ Pic at a few 100 Myr (Barrado y Navascués et al. 1999 and refs. therein). The youth of $\beta$ Pic was soon firmly established by Barrado y Navascués et al. (1999), who honed lists of stars potentially comoving with $\beta$ Pic and identified just three M star "survivors," all of age $\sim 20$ Myr. This work constituted the first identification of the $\beta$ Pic Moving Group ( $\beta$ PMG) - its original membership, like that of the original TWA, could be counted on one hand. Not long thereafter, the wider availability and improved application of space velocity data would begin to dramatically expand the known membership of the $\beta$ PMG (Zuckerman et al. 2001).

At the risk of stating the obvious, the general approach pioneered by Zuckerman (e.g., Zuckerman \& Webb 2000; Zuckerman et al. 2001), Song (e.g., Song et al. 2002), Torres (Torres et al. 2006), and a few other investigators - i.e., selecting candidate young stars on the basis of proper motions and/or X-ray fluxes, and following up spectroscopically to confirm signatures of youth and obtain $U V W$ velocities - has been refined and improved

$\dagger$ This (traceback-based) age estimate seems relatively robust (Ducourant et al. 2014). However, given the many important caveats regarding ages of NYMGs that have been raised at this meeting (see, e.g., reviews by Mamajek and Bell), the age of the TWA certainly bears revisiting via other methods.

$\ddagger$ With apologies, and thanks, to David Wilner. 
tremendously over the past $\sim 15$ years. In particular, statistically rigorous treatments of stellar kinematics (e.g., Malo et al. 2013) have replaced the original, painstaking, "byhand" searches of the Hipparcos and Tycho catalogs, and the Galex UV sky survey data have replaced the RASS as a superior means to select active (hence, possibly young) latetype stars in the field (Shkolnik et al. 2011; Rodriguez et al. 2013). Thus, the techniques first brought to bear to identify and confirm members of the TWA and $\beta$ PMG have now been successfully applied to identify (and/or expand the memberships of) another dozen or so established and candidate NYMGs (see, e.g., Zuckerman et al. 2011 and review by Mamajek in these Proceedings).

\section{The impact of nearby young moving group studies}

I summarize here three recent examples of studies involving $\beta \mathrm{PMG}$ members that highlight the utility of studies of NYMGs and their members for purposes of improving our understanding of pre-main sequence (pre-MS) stellar evolution, evolved protoplanetary disks, and young exoplanets.

\subsection{Understanding early stellar evolution}

The comprehensive study of the $\beta$ PMG by Mamajek \& Bell (2014) well illustrates how a NYMG can serve as a testbed both for the astrophysics of pre-main sequence stars and techniques for age-dating stars and stellar associations. Mamajek \& Bell compiled and critically compared a dozen $\beta \mathrm{PMG}$ age determinations involving roughly a halfdozen different techniques, including placement on pre-MS star isochrones, kinematic traceback, Li depletion, and variants thereof. They conclude that the age of the $\beta \mathrm{PMG}$ should be revised upwards, from the widely quoted $~ 12$ Myr (Zuckerman et al. 2001; Torres et al. 2006) to $\sim 23 \mathrm{Myr}$ (which is, ironically, closer to the original estimate by Barrado y Navascués et al. 1999). However, this refinement in the age of the $\beta \mathrm{PMG}$ is perhaps less interesting than the conclusion by Mamajek \& Bell (2014) that, at least in application to the $\beta$ PMG stars, the Li depletion boundary and isochronal age estimation techniques are superior to kinematic methods of age determination. Given the interesting, fundamental problems in stellar astrophysics that are inherent in the former two methods (as highlighted in talks by Bell, Song, Somers and others at this meeting), it is clear that the tests of Li depletion boundary and isochronal age determinations offered by studies of NYMGs will become increasingly important, moving forward.

\subsection{Investigating (evolved) protoplanetary disks}

Though it remains understudied, especially relative to TW Hya, the $\beta$ PMG member system V4046 Sgr is nearly as close $(D \sim 73 \mathrm{pc})$ and, arguably, at least as interesting $\dagger$. This hierarchical multiple system features a close, near-equal-components, solar-mass binary that is orbited by, and actively accreting from, a molecule-rich circumbinary disk. The disk is surprisingly extensive and massive (outer $\mathrm{CO}$ radius $\sim 350 \mathrm{AU}$ and estimated $\mathrm{H}_{2}$ mass $\sim 0.09 M_{\odot}$, Rosenfeld et al. 2013; Fig. 3.2, left panel) given the advanced age of V4046 Sgr and, hence, potentially affords the opportunity to study late stages in the process of planet formation around a close binary. Recently, Rapson et al. (2015) used the new Gemini Planet Imager (GPI) to obtain near-infared coronagraphic/polarimetric (scattered-starlight) images of the innermost disk regions (Fig. 3.2, right panel). Thanks to the combination of GPI's exquisite performance and the proximity of V4046 Sgr, these

$\dagger$ For a brief overview of V4046 Sgr, see the May 2013 Star Formation Newsletter: http://www.ifa.hawaii.edu/ reipurth/newsletter/newsletter245.pdf. 


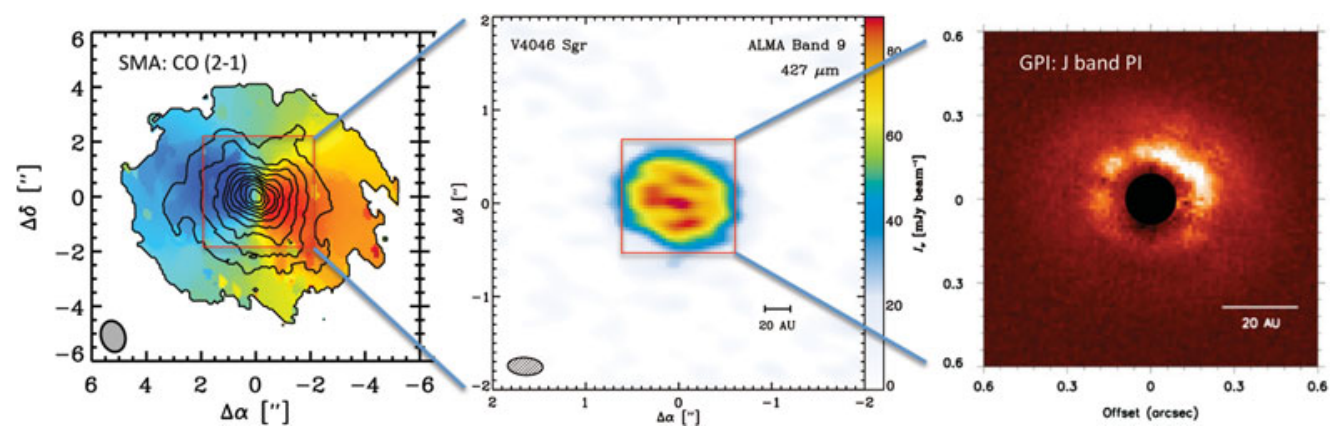

Figure 1. The V4046 Sgr disk as imaged in CO line emission by the Submillimeter Array (left; from Rosenfeld et al. 2013), in $427 \mu \mathrm{m}$ continuum emission by ALMA during Early Science (Cycle 0) operations (center; from Andrews et al., in prep.), and in polarized intensity at 1.25 $\mu \mathrm{m}$ by the Gemini Planet Imager in its coronagraphic/polarimetric mode (right; from Rapson et al. 2015).

images probe the structure of the dust disk to within $\sim 7$ AU of the central binary, at a jaw-dropping $\sim 3$ AU resolution. The GPI imaging reveals concentric rings of submicronsized dust grains that are contained within the ring of submm-sized grains responsible for thermal emission in submm continuum imaging. The structures seen in these (GPI and ALMA) images of V4046 Sgr (Fig. 3.2) are strikingly similar to those seen in synthetic images derived from simulations describing newborn planets clearing gaps within a protoplanetary disk (see, e.g., Fig. 7 in Dong et al. 2015).

\subsection{Discovering and characterizing young exoplanets}

Because young gas giant planets are self-luminous in the infrared, NYMG members offer the best targets for extreme adaptive optics imaging of giant exoplanets. Indeed, direct imaging of nearby, young stars represents the only means that will be available in the near future to detect exoplanets in wide ( $>5 \mathrm{AU}$ ) orbits around their host stars (see, e.g., review by Chauvin in these Proceedings). This potential for NYMG members especially those hosting dusty debris disks - to yield direct-imaging detections of massive exoplanets is, of course, amply demonstrated by the detection and subsequent intensive study of $\beta$ Pic b (e.g., Bonnefoy et al. 2014). With GPI and SPHERE now having demonstrated their capabilities, we appear to be on the verge of a trove of such directimaging discoveries of planets orbiting NYMG member stars.

\section{Acknowledgements}

JHK's research on young stars near Earth is supported by NSF grant AST 1108950 and NASA Astrophysics Data Analysis Program grant NNX12AH37G to RIT.

\section{References}

Barrado y Navascués, D., Stauffer, J. R., Song, I., \& Caillault, J.-P. 1999, ApJL, 520, L123

Bonnefoy, M., Marleau, G.-D., Galicher, R., et al. 2014, A\&\&A, 567, L9

de la Reza, R., Torres, C. A. O., Quast, G., Castilho, B. V., \& Vieira, G. L. 1989, ApJL, 343, L61

Dong, R., Zhu, Z., \& Whitney, B. 2015, ApJ, 809, 93

Ducourant, C., Teixeira, R., Galli, P. A. B., et al. 2014, A\& A, 563, A121

Gregorio-Hetem, J., Lepine, J. R. D., Quast, G. R., Torres, C. A. O., \& de La Reza, R. 1992, $A J, 103,549$

Henize, K. G. 1976, ApJS, 30, 491

Herbig, G. H. 1978, in Problems of Physics and Evolution of the Universe, 171 
Jura, M., Malkan, M., White, R., et al. 1998, ApJ, 505, 897

Kastner, J. H., Zuckerman, B., Weintraub, D. A., \& Forveille, T. 1997, Science, 277, 67

Malo, L., Doyon, R., Lafrenière, D., et al. 2013, ApJ, 762, 88

Mamajek, E. E. \& Bell, C. P. M. 2014, MNRAS, 445, 2169

Rapson, V. A., Kastner, J. H., Andrews, S. M., et al. 2015, ApJL, 803, L10

Rodriguez, D. R., Zuckerman, B., Kastner, J. H., et al. 2013, ApJ, 774, 101

Rosenfeld, K. A., Andrews, S. M., Wilner, D. J., Kastner, J. H., \& McClure, M. K. 2013, ApJ, 775,136

Rucinski, S. M. \& Krautter, J. 1983, A\&\&A, 121, 217

Shkolnik, E. L., Liu, M. C., Reid, I. N., Dupuy, T., \& Weinberger, A. J. 2011, ApJ, 727, 6

Song, I., Bessell, M. S., \& Zuckerman, B. 2002, AËA, 385, 862

Stauffer, J. R., Hartmann, L. W., \& Barrado y Navascues, D. 1995, ApJ, 454, 910

Torres, C. A. O., Quast, G. R., da Silva, L., et al. 2006, A\&A, 460, 695

Webb, R. A., Zuckerman, B., Platais, I., et al. 1999, ApJL, 512, L63

Zuckerman, B., Forveille, T., \& Kastner, J. H. 1995, Nature, 373, 494

Zuckerman, B. \& Webb, R. A. 2000, ApJ, 535, 959

Zuckerman, B., Song, I., Bessell, M. S., \& Webb, R. A. 2001, ApJL, 562, L87

Zuckerman, B., Rhee, J. H., Song, I., \& Bessell, M. S. 2011, ApJ, 732, 61 